\title{
Harlequin Ichthyosis: Folklore of Demon
}

\section{Sunil O *1, Trinesh Gowda M S .}

${ }^{*}$ Department of Anatomy, Mandya Institute of Medical Sciences, Mandya, Karnataka, India.

2 Department of Anatomy, Mandya Institute of Medical Sciences, Mandya, Karnataka, India.

\section{ABSTRACT}

Background: Harlequin ichthyosis ( $\mathrm{HI})$ is a severe and rare autosomal recessive congenital ichthyosis, characterized by severe hyperkeratosis, extensive fissuring and massive, diamond-shaped scales which limit the child's movements. The term harlequin comes from the baby's facial expression and diamond-shaped pattern of the scales like the 17th century entertainers, harlequins. The underlying genetic abnormality has been identified as a mutation in the lipid-transporter gene ABCA 12 on chromosome 2q35.

Aim and objectives: To provide a comprehensive knowledge of severe forms of congenital ichthyosis, the harlequin ichthyosis and abolish the superstitious notion regarding the appearance of the neonate.

Materials and Methods: The present report is case of premature girl baby delivered at MIMS, Mandya. The neonate presented plaques of rigid fixed skin, separated by deep red fissures, facial features were obliterated by thickened skin, undeveloped nose and pinna, severe ectropion and eclabium. Intensive care was given and survival was prolonged by systemic retinoids. The neonate succumbed to respiratory failure few days later.

Results and conclusion: The present study gives a comprehensive knowledge of harlequin ichthyosis and provides emphasis on sensitization of the disease to prevent any misconception or superstition. Also lays emphasis on Genetic counseling of the couple to prevent further occurrences.

KEY WORDS: Eclabium, Ectropion, Harlequin ichthyosis.

Corresponding Author: Dr. Sunil O, Department of Anatomy, Mandya Institute of Medical Sciences, Mandya, Karnataka, India. E-Mail: sunilobulesh13@gmail.com

Access this Article online

Quick Response code

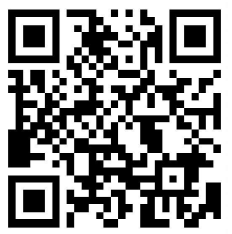

DOI: 10.16965/ijar.2021.191
Journal Information

International Journal of Anatomy and Research

ISSN (E) 2321-4287 | ISSN (P) 2321-8967

https://www.ijmhr.org/ijar.htm

DOI-Prefix: https://dx.doi.org/10.16965/ijar

Article Information

Received: 16 Nov 2021

Peer Review: 17 Nov 2021

Accepted: 03 Jan 2022

Published (O): 05 Mar 2022

Published (P): 05 Mar 2022

\section{INTRODUCTION}

The birth of a newborn is an important milestone for parents. the well-being of the newborn decides the emotional stability of the couple. Any disease or disorder will have physical, mental and financial restraint on the family. Harlequin ichthyosis (HI) is a rare genetic skin disorder, with prevalance of 1 in 300,000 newborns [1]. It is an autosomal recessive congenital ichthyosis, characterized by severe hyperkeratosis, extensive fissuring and massive, diamond-shaped scales which limit the child's movements. The eyes, mouth, and ears are commonly affected due to the tightening of the skin. Further complications include difficulty with breathing and eating, dehydration, infection, thermoregulation, and can eventually lead to death $[2,3]$. The term harlequin comes from the baby's facial expression and diamond-shaped pattern of the scales like the 17th century entertainers, harlequins [4].

\section{Aim and objectives:}

To provide a comprehensive knowledge of severe forms of congenital ichthyosis, the harlequin ichthyosis and abolish the superstitious notion regarding the appearance of the neonate. 


\section{CASE REPORT}

The present case report is case of premature girl baby delivered at Mandya Institute of Medical Sciences, Mandya to a non-consanguineous married couple. The baby was prematurely delivered by normal vaginal delivery; birth weight recorded was $1.8 \mathrm{~kg}$. The mother was 2 nd para, last pregnancy was uneventful. The prenatal and perinatal period of the present pregnancy was uneventful with normal anomaly scan. The news of the birth of newborn with such skin disorder led to speculation of child with demon or monster like appearance. The neonate presented with plaques of rigid fixed skin, separated by deep red fissures, facial features were obliterated by thickened skin, yellow in color, underdeveloped flattened nose with absence of external nostrils and flattened pinna, severe ectropion of both the palpebral fissures and eclabium involving both upper and lower lip.

The upper limbs showed flexion at the elbow and wrist joint. the lower limbs showed flexion at the hip and knee joints. Both the hands and feet were edematous due to the constriction ring produced by the thickened scaly skin, movements were restricted. No other congenital defects were noted, which helped to differentiate the present case from stiff case syndrome, SAM syndrome, CHILD syndrome, Netherton syndrome and Neu-Laxouva syndrome. Intensive care was given in neonatal intensive care unit. The newborn was placed in a humidifier to prevent dehydration and hypothermia. The vitals were closely monitored and survival was prolonged by systemic retinoids $1 \mathrm{mg} / \mathrm{kg}$ body weight. On the second day of admission, the skin fissures were noticed to be oozing serous fluid which gradually progressed to serosanguineous exudates. Basic investigations were done, results showed deranged electrolytes with hyponatremia ( $\mathrm{Na}-116 \mathrm{mmol} / \mathrm{L})$, hypokalemia ( $\mathrm{K}-2.8 \mathrm{mmol} / \mathrm{L})$ and severe acidosis ( $\mathrm{HCO} 3-10 \mathrm{mmol} / \mathrm{L}$ ). Complete blood count was also altered, PCV was $28 \%$ and platelet was less than $10,000 / \mathrm{mm} 3$. Blood culture yielded no growths. Parents declined autopsy. The neonate succumbed to respiratory failure few days later unfortunately.

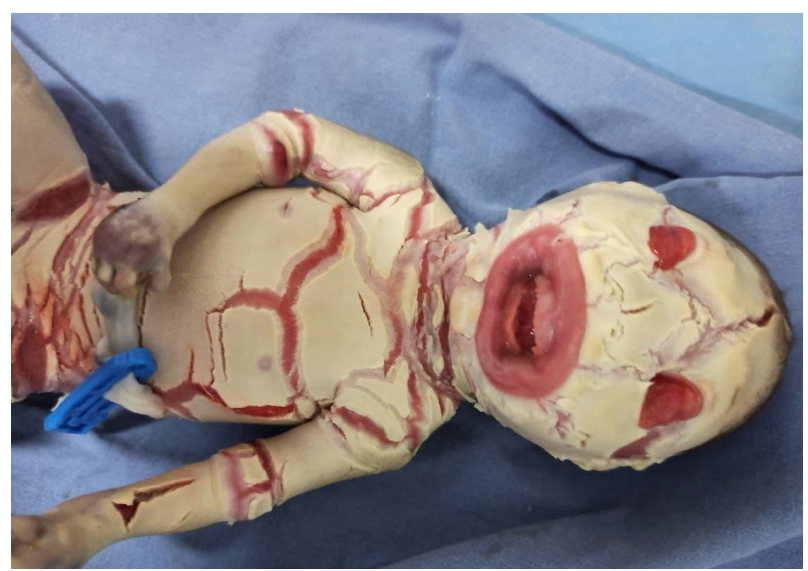

Fig. 1: Thick rigid skin, yellow in color.

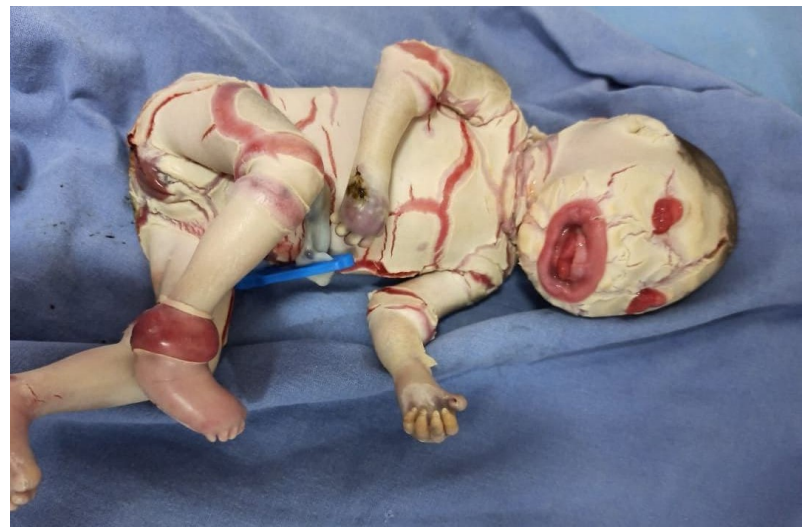

Fig. 2: Bilateral ectropion, eclabium of both upper and lower lib, hypoplastic pinna and external nose.

\section{DISCUSSION}

Ichthyoses are inborn keratinizaton disorders defined by universal scaling occurring over the entire body, some manifest at birth and some at birth [5]. The term harlequin comes from the baby's facial expression and diamondshaped pattern of the scales like the 17th century entertainers, harlequins. Harlequin refers to a stock character of the Italian commedia dell'arte. The character began in the 16thcentury as an unscrupulous comic servant, later became a faithful valet involved in amorous exploits, and ultimately evolved into a clown like figure engaging in good-natured joking and amusing tricks [4-7]. This disorder is caused by a mutation in the ABCA12 gene on chromosome $2 q 35$. This gene makes proteins which play an essential role in the normal development of skin cells. The protein helps transport molecules, mainly lipids (fat), in the epidermis of the skin. The lipids in the epidermis help prevent water loss. As the mutation causes a change in the lipid transportation system, the skin is unable to form as a protective barrier. As a result, the skin 
becomes thick, scaly and mobility is compromised $[2,8]$. Loss of ability of the skin to form a protective barrier and thickening, hyper keratosis can occur. Neonates are more prone to sepsis, dehydration and thermoregulation is compromised [8].

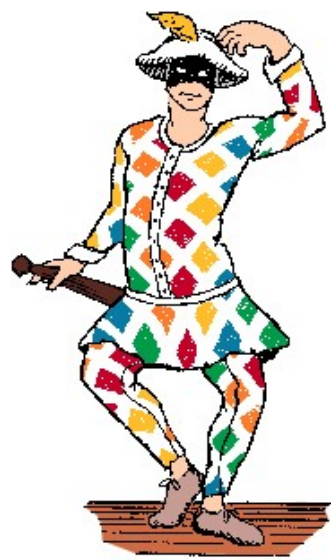

Fig. 3: Illustration of harlequin [7].

Babies that survive past infancy develop skin changes that come from the same family of genetic skin disorders, known as non-bullous congenital ichthyosiform erythroderma [9]. As a result, the skin becomes red and is usually itchy or painful [10]. The tightening of the skin commonly affects the eyes and ears resulting in vision problems, such as developing cataracts, and deafness may also occur. Respiratory problems are the most common in the early stages of Harlequin Ichthyosis due to the constriction of the skin and can eventually lead to respiratory failure and death $[9,10]$. Diagnosis is through clinical appearance and physical examination after birth. Routine ultrasonography show signs of lesions, such as eversion of the lips and eyelids. Accurate diagnosis of Harlequin Ichthyosis is by DNA analysis for the mutation of the ABCA12 gene. Women with history of previous pregnancies with harlequin babies are advised to have prenatal testing such as Amniocentensis and Chorionic villus sampling [11].

Those surviving the neonatal period, lives with a chronic and recurring skin disease. Since the neonatal care has greatly advanced over the years the survival of the baby is achieved to a greater extent. After a baby has been born with Harlequin Ichthyosis, they are sent to the neonatal intensive care unit to prevent complications. Broad spectrum antibiotics are used to keep the infections at bay in the early neonatal stages. Patients with delayed fine and gross motor skills essentially require occupational or speech-language therapy $[9,11]$. Those affected will have to keep their skin moist by applying skin softening creams, this will help prevent cracking and reduce the risk of infections, mild keratolytic therapies to remove excess skin.

Recent advancement in treatments include oral retinoids that have shown positive changes such as softening of the skin and reducing contracture thereby improving limb mobility. Screening for the disease may help couple who carry recessive gene for the trait. Parents who are such carriers for the disorder have a $25 \%$ chance of transmitting the disease per pregnancy $[2,11]$. Gene therapy is a possibility for some couples. However, there are risks of further genetic mutations. Advancements in intensive neonatal care have positively impacted both short and long-term outcomes. Dr. Paller and fellow ichthyoses researchers have analyzed the linkages between TH-17 and IL-23 pathways, which are involved in inflammation and epidermal pathologies. Their findings suggest that the immune profile of ichthyosis is like psoriasis, and psoriasis treatments may be used for patients affected by ichthyosis [11].

Sociocultural stigma still persists and the affected neonates are being referred as monster or demon baby.

\section{CONCLUSION}

Harlequin ichthyosis, is a severe form of congenital ichthyosis which has high mortality and morbidity. Supportive care from a multi-disciplinary team is required for effective management. The affected families need to be carefully followed up and supported. There is also a need for awareness creation concerning the disease in both health care workers and the populace with the development of national guidelines. Emphasis must be laid down to prevent stigmatization of such neonates.

\section{ABBREVIATIONS}

ABCA12 - ATP binding cassette sub-family 12 MIMS - Mandya Institute of Medical Sciences HI - Harlequin ichthyosis 


\section{Author Contributions:}

Sunil $\mathbf{O}$ - Collecting the data and done the field work ORCID: https://orcid.org/0000-0001-5916-7161

Trinesh Gowda MS- formulation the design of study and guidance for final manuscript drafting.

\section{REFERENCES}

[1]. Rathore S, David LS, Beck MM, Bindra MS, Arunachal G. Harlequin ichthyosis: Prenatal diagnosis of a rare yet severe genetic dermatosis. J Clin Diagn Res. 2015;9(11):QD04-QD06.

[2]. National Organization for Rare Disorders. Ichthyosis, harlequin type. https://rarediseases.org/rarediseases/ichthyosis-harlequin-type/

[3]. Glick JB, Craiglow BG, Choate KA, Kato H, Fleming $\mathrm{RE}$, Siegfried E, \& Glick SA. Improved management of harlequin ichthyosis with advances in neonatal intensive care. Am Aced Pediatrics. 2017;139(1).

[4]. Sundaramoorthy Srinivasan. Expecting the most unexpected - a harlequin baby! A case report and literature analysis. Our Derm online. 2012.

[5]. Krug M, Oji V, Traupe H, Berneburg M. IchthyosesPart 1: Differential diagnosis of vulgar ichthyoses and therapeutic options. J. Dtsch. Dermatol. Ges. 2009; 7: 511-519.
[6]. Krug M, Oji V, Traupe H, Berneburg M. IchthyosesPart 2: Congenital ichthyoses. J. Dtsch. Dermatol. Ges. 2009; 7: 577-588.

[7]. Merriam-Webster: History of Harlequin.

[8]. Available from: https://www.merriam-webster.com /dictionary/harlequin [Accessed 15th Nov 2021].

[9]. U.S. National Library of Medicine. ABCA12 gene. https://ghr.nlm.nih.gov/gene/ABCA12\#conditions. Reviewed July 2017.

[10]. Rajpopat, S et al. Harlequin ichthyosis: a review of clinical and molecular findings in 45 cases. Arch Dermatol. 2011;147(6):681-86.

[11]. Orphanet. Congenital non-bullous ichthyosiform erythyroderma.

[12].https://www.orpha.net/consor/cgi-bin/ OC_Exp.php?Ing=en\&Expert=79394. Updated Jan 2012.

[13]. Paller, A et al. An IL-17-dominant immune profile is shared across the major orphan forms of ichthyosis. J Allergy Clin Immunol. 2017;139(1):152-65.

How to cite this article:

Sunil O, Trinesh Gowda M S. Harlequin Ichthyosis: Folklore of

Demon. Int J Anat Res 2022;10(1):8226-8229. DOI: 10.16965/

ijar.2021.191 